\title{
2 Research Square \\ Application of Estrogen for the Treatment of Stress Urinary Incontinence in Mice
}

\section{Lu Li}

Renmin Hospital of Wuhan University

\section{Shasha Hong}

Renmin Hospital of Wuhan University

\section{Yang Li}

Renmin Hospital of Wuhan University

\section{Xiang Li}

Renmin Hospital of Wuhan University

\section{Lian Yang}

Renmin Hospital of Wuhan University

Jianfeng Liu

Renmin Hospital of Wuhan University

Li Hong ( $\nabla$ dr_hongli@whu.edu.cn )

Wuhan University Renmin Hospital

\section{Research Article}

Keywords: Estrogen, Extracellular Matrix, Stress Urinary Incontinence

Posted Date: January 24th, 2022

DOI: https://doi.org/10.21203/rs.3.rs-1274509/v1

License: (c) (i) This work is licensed under a Creative Commons Attribution 4.0 International License.

Read Full License

Version of Record: A version of this preprint was published at Archives of Gynecology and Obstetrics on February 16th, 2022. See the published version at https://doi.org/10.1007/s00404-022-06435-1. 


\section{Abstract}

Background The etiology of stress urinary incontinence (SUI) is complex and diverse among women, with past studies having demonstrated that estrogen deficiency is an important cause of pelvic floor muscle atrophy and urethral degeneration. We comprehensively investigated the effects of estrogen in the treatment of SUI in female mice at cellular and animal levels.

Methods L929 cell mechanical injury model was established by four point bending device, and SUI mouse model was established by vaginal dilation method commonly used to simulate labor injury. After treatment, the expressions of collagen I, collagen III, elastin, TIMP-1, TIMP-2, MMP-2 and MMP-9 were detected, the leak point pressure (LPP) and abdominal leak point pressure (ALPP) of mice in each group were detected, and the effect of estrogen on extracellular matrix remodeling of mouse urethra and anterior vaginal wall was observed from the histological level.

Results These results revealed that an appropriate amount of estrogen can promote the expression of collagen I, collagen III, elastin, TIMP-1, and TIMP-2, decrease the expression of MMP-2 and MMP-9, and maintain the dynamic balance of MMPs/TIMPs at both cellular and animal levels. Meanwhile, we determined that estrogen can increase the LPP and ALPP values of SUI mice. The collagen fibers' content in the mice treated with estrogen was significantly greater than in the control group mice.

Conclusions The estrogen may alleviate the symptoms of SUI by reconstituting ECM, toward laying a solid foundation for the further exploration of estrogen therapy.

\section{Introduction}

Stress urinary incontinence (SUI) is a usual disease affecting women, which refers to a type of pelvic floor dysfunction disease wherein urine leaks involuntarily from the external urethra at enhanced abdominal pressure due to activities such as coughing, exercising, laughing, or sneezing ${ }^{1,2}$. SUI can lead to physical, psychic, and social adverse effects, thereby seriously affecting the quality of life ${ }^{3}$. The etiology of SUI is complex and diverse for women, including obesity, vaginal delivery, aging, decreased ovarian function, and estrogen deficiency ${ }^{4-7}$. Previous studies have indicated that estrogen deficiency is an important cause of pelvic floor muscle atrophy and urethral degeneration ${ }^{8}$. The Phase III clinical trial of the estrogen receptor blocker levormeloxifene revealed that its main side effect was to induce SUI ${ }^{9}$. In addition, the incidence rate of postmenopausal SUI was significantly higher ${ }^{10}$.

In recent years, in parallel with the development of the social economy, the diagnosis and treatment of SUI have attracted more and more attention from the medical profession, with the development of newer diagnoses and treatment methods. Presently, the treatment of mild to moderate SUI often uses conservative therapies, such as biofeedback therapy ${ }^{11}$, pelvic floor muscle training ${ }^{12}$, and drug therapy such as topical estrogen therapy ${ }^{13}$. Severe SUI treatment is usually based on surgical methods, including mesh vaginal surgery ${ }^{14}$, placement of synthetic slings ${ }^{15}$, local injection of bulking agents, and 
implantation of artificial urinary sphincters ${ }^{16,17}$. For patients with mild to moderate symptoms, topical estrogen therapy is the preferred approach owing to its convenience and fewer side effects. However, for a long time, the basic research of estrogen in the treatment of SUI is relatively lacking.

Extracellular matrix (ECM) is an important portion of the connective tissues of the pelvic floor. It is a complex long-chain protein mixture, composed of collagen, elastin, and proteoglycan ${ }^{18}$. Previous studies have confirmed that ECM remodeling is an important association in the pathogenesis of SUI ${ }^{19}$. It has been found that estrogen affects the transcription and expression of collagen, which are the main component of $\mathrm{ECM}^{20}$. Estrogen replacement therapy (ERT) has been reported to reshape collagen in the urogenital tissues of postmenopausal women by changing its quantity and quality ${ }^{21}$. Animal experiments have proven that estrogen can increase the composition of loose connective tissues rich in blood vessels in the urethra ${ }^{22}$, and, at the same time, increase the distribution density of sympathetic nerves in the pelvic floor tissues and regulate neurotrophy ${ }^{23}$. Therefore, the supplementation of exogenous estrogen in postmenopausal SUI patients can effectively alleviate the symptoms of $\mathrm{SUI}^{24}$.

Although the application of estrogen as a conservative treatment is clinically effective in the treatment of $\mathrm{SUI}^{24}$, the basic research of estrogen in the treatment of SUI is relatively lacking. Meanwhile, reports on the remodeling of ECM caused by estrogen are not systematic. Therefore, in this article, according to our previous experience ${ }^{25}$, we constructed the animal model of stress urinary incontinence by vaginal dilation, and correspondingly, we constructed the mechanical injury model of $L 929$ fibroblasts $^{26}$. We have described the application of topical estrogen to treat SUI from the animal and cellular level to investigate the reasons affecting the efficacy of topical estrogen for SUI, the mechanism by which estrogen affects the expression of ECM, and then pave the way for the mechanism of treatment effects.

\section{Materials And Methods}

\subsection{Cell Experimental Design and Cell Culture}

Mouse L929 fibroblasts were purchased from Procell Life Science \& Technology Co., Ltd. (Wuhan, China). Fibroblasts were fostered in Dulbecco's modified Eagle's medium (DMEM) (Procell Life Science \& Technology Co., Ltd.), (supplemented with $4.5 \mathrm{~g} / \mathrm{L}$ D-glucose, without Phenol red indicator) with $10 \%$ fetal bovine serum (FBS; Gemini Bio-Products, California, USA) and 1\% antibiotics (100 KU/mL penicillin G, $100 \mathrm{mg} / \mathrm{mL}$ streptomycin; Genom Biotech Ltd.) in an incubator at $37^{\circ} \mathrm{C}$ under $5 \% \mathrm{CO}_{2}$ atmosphere.

In order to explore the expression of ECM without or with estrogen therapy before and after cell stress loading, we categorized the cells with the same growth status into four groups: normal cells without estrogen therapy (Group C), normal cells with estrogen therapy (Group E2), cells that had undergone cyclic mechanical strain with estrogen therapy (Group S+E2), and cells that had undergone cyclic mechanical strain without estrogen therapy (Group S). For cells treated with estrogen, the concentration of estrogen was $10^{-5} \mathrm{nmol} / \mathrm{L}$, which was based on cell proliferation and apoptosis experiments. These 
four groups of cells were cultured in an incubator for $4 \mathrm{~h}$ after their respective treatments, followed by collection in 6-well plates for subsequent experiments.

\subsection{Cyclic Mechanical Strain}

The L929 cells were collected on four-point bending cell strain gauges (Miracle Technology Co. Ltd., Chengdu, China) at a density of $1 \times 10^{5}$ cells $/ \mathrm{mL}$, and 2-mL cell suspension from this was uniformly spread on each of the strain gauges. Next, 4 pieces of stress gauges were laid each time, and only 2 of them were subjected to mechanical stress loading. The cells were continuously cultured in an incubator with $5 \% \mathrm{CO}_{2}$ at the temperature of $37^{\circ} \mathrm{C}$ for $24 \mathrm{~h}$ to prepare the cell slide. While the cells adhered firmly and grew up to a sub-fusion state, the slide was placed in the strain state containing $65-\mathrm{mL}$ of complete culture medium and then stretched for mechanical strain loading, as shown in Fig. 1(b). The parameters of the four-point bending facility parameters (Miracle Technology Co. Ltd., Chengdu, China) were adjusted to a frequency of $1 \mathrm{~Hz}$ for $4 \mathrm{~h}$ and the cells were subjected to $5333 \mu$ as described in our previous research, using $0 \mu$ strain as the control strain ${ }^{27}$. At the end of the cyclic mechanical strain, the cells on the slide were cultured for another $4 \mathrm{~h}$, as shown in Fig. 1(c). Then, all the cells were collected to detect the corresponding indicators.

\subsection{Cell Proliferation}

$\beta$-Estradiol (E8875; Sigma) was dissolved in anhydrous ethanol to a concentration of $1 \mathrm{M}$, which was further diluted in DMEM (4.5 g/L D-glucose, without Phenol red indicator) to make the final concentration of $\beta$-estradiol to $10^{-3}, 10^{-4}, 10^{-5}, 10^{-6}, 10^{-7}$, and $10^{-8} \mathrm{nmol} / \mathrm{L}$, respectively. The $\mathrm{L} 929$ cells were uniformly cultured in 96-well plates at the density of 5000 cells per well and fostered in a medium containing different concentrations of estradiol $(100 \mu \mathrm{L}$ medium per well. The proliferation of L929 cells was investigated by using the Cell Counting Kit-8 (CCK-8; Dojindo Molecular Technologies, Inc., Shanghai, China) for $24 \mathrm{~h}$. The medium of each well was discarded, replaced with a mixture containing $90 \mu \mathrm{L}$ serum-free medium and $10 \mu \mathrm{L}$ of CCK-8 reagent to each well. After $2 \mathrm{~h}$, the absorbance was measured by spectrophotometer at $450 \mathrm{~nm}$ (5 holes in each group).

\subsection{Western Blotting}

After applying the design-based treatment, the proteins were successfully obtained from L929 cells, the urethra, and the anterior vaginal wall of mice with RIPA lysate supplemented with phenylmethanesulfonyl fluoride (PMSF, 1 mM; Servicebio, Wuhan, China). Western blotting was performed as described previously ${ }^{27}$. The antibodies used are listed below: anti-Collagen type I antibody (1:1000, Abcam, ab88147), anti-collagen type III antibody (1:500, Abcam, ab7778), anti-Elastin antibody (1:1000, Abcam, ab2173560), anti-MMP-2 antibody (1:1000, Abcam, ab86607), anti-MMP-9 antibody (1:1000, Abcam, ab228402), anti-TIMP-1 antibody (1:500, Santa, sc-21734), anti-TIMP-2 antibody (1:500, Santa, sc21735), and anti-GAPDH antibody (1:1000, Abcam, ab8245).

\subsection{Quantitative Real-Time Polymerase Chain Reaction (Q- PCR)}


Q-PCR was performed to investigate the expression of related genes in this experimental design. The reaction was performed as described elsewhere ${ }^{27}$. All the primers used in the study were obtained from Sangon Biotech (Shanghai, China; listed in Table 1).

Table 1

A list of primers used for the quantitative real-time polymerase chain reaction in this study.

\begin{tabular}{|llll|}
\hline Gene name & Gene ID & Primer sequence (5'-3') & Amplicon size (bp) \\
\hline M-GAPDH & NM_008084.3 & F: TGAAGGGTGGAGCCAAAAG & 227 \\
& & R: AGTCTTCTGGGTGGCAGTGAT & \\
M-COL1A1 & NM_007742.4 & F: CTGACTGGAAGAGCGGAGAG & 116 \\
M-Col3a1 & NM_009930 & F: CTCAAGAGTGGAGAATACTGGGTT & 298 \\
& & R: GGTATGTAATGTTCTGGGAGGC & \\
M-MMP2 & NM_008610.2 & F: GAATGCCATCCCTGATAACCT & 127 \\
& & R: GCTTCCAAACTTCACGCTCTT & \\
\hline M-MMP9 & NM_013599.3 & F: AAGGGTACAGCCTGTTCCTGGT & 149 \\
& & R: CTGGATGCCGTCTATGTCGTCT & \\
M-TIMP-1 & NM_053819.1 & F: CCAGAAATCATCGAGACCACC & 201 \\
& & R: ATTTCCGTTCCTTAAACGGC & \\
\hline M-TIMP-2 & NM_011594.3 & F: GACACGCTTAGCATCACCCA & 140 \\
& & R: TCTGTGACCCAGTCCATCCAG & \\
\hline M-Elastin & NM_007925.4 & F: ACCCACAGGACAAGGAAATCA & 177 \\
& & R: CCCACAAAGAAGAAGCACCA & \\
\hline
\end{tabular}

\subsection{Animal Experiment Design and Treatment}

All our animal experimental protocols and implementations were conducted based on the institutional guidelines of the Institutional Review Committee and authorized by the Ethics Committee of the Institutional Animal Care and Use Committee of Wuhan University People's Hospital (20210306).

We purchased wild-type C57BL/6 female mice (8-9-week-old) from the Center for Animal Experiment of Wuhan University. A total of 60 wild-type C57BL/ 6 female mice were stochastically separated into four groups, as detailed here: control group (Group C), estrogen-alone group (Group E2), vaginal dilatation group (Group VD), and vaginal dilation with estrogen group (Group VD+E2). There were 15 mice in each 
group. The groups VD and VD+E2 were subjected to vaginal dilatation on the same day. From the second day of vaginal dilation, conjugated estrogen cream (CEEs; Xinjiang Xinziyuan Bio-Pharmaceutical Co. Ltd, Yili, China) was applied to the vagina of the mice in groups E2 and VD + E2 using a 1-mL syringe. The calculation of vaginal CEE dose was based on the comparison of body weight and human dose, as mentioned in a previous study ${ }^{28}$. The usual vaginal maintenance dose $(1 \mathrm{~g}$ cream) for a $60-\mathrm{kg}$ woman could produce $625 \mu \mathrm{g}$ of CEEs. By weight comparison, the CEE dose for a 20-g animal was found to be $0.21 \mu \mathrm{g}$.

After 7 days of continuous administration of CEE to the mice in the two groups, the suprapubic canal was implanted in all mice belonging to the four groups, and the leakage point pressure (LPP) and abdominal pressure LPP (ALPP) were measured on the same day. Then, the anterior vaginal wall and the urethra of inanimate mice were obtained for western blotting and section staining.

\subsection{Masson trichrome staining}

A portion of the animal samples was intercalated into paraffin, sliced into transverse sections of thickness $4 \mu \mathrm{m}$, and stained with the Masson Kit HT15 (Sigma, USA) following the manufacturer's protocol. The mean optical density (MOD) value gathered by the ImageJ software was considered as the quantitative index to assess the content of collagen fibers.

\subsection{Statistical analysis}

All data were expressed as mean \pm standard deviation. Statistical analysis was conducted with the GraphPad Prism software version 8 (San Diego, CA, USA). One-way analysis of variance (ANOVA) was employed for several comparisons in three or more groups. $\mathrm{P}<0.05$ was regarded as statistically significant.

\section{Results}

\subsection{Estradiol promotes the proliferation of L929 fibroblasts}

The proliferation effect of estradiol at different concentrations on L929 cells was detected by the CCK8 assay. As shown in Fig. 1(a), $\beta$-estradiol had an increasingly significant proliferation effect on the L929 cells with increasing concentration. Specifically, at the concentration of $10^{-5} \mathrm{nmol} / \mathrm{L}$, the $L 929$ cells showed the maximum proliferation effect. These data suggest that $10^{-5} \mathrm{nmol} / \mathrm{L}$ concentration is the optimal concentration for $L 929$ fibroblasts proliferation. Therefore, we selected $10^{-5} \mathrm{nmol} / \mathrm{L}$ as the optimal concentration of $\beta$-estradiol to treat L929 fibroblast for the subsequent experiments.

\subsection{The promoting effect of estradiol on the expression of ECM for L929 fibroblasts}

ECM is a complex long-chain protein mixture composed of collagen, elastin, and proteoglycan. Among these constituents, collagen I and collagen III are the main collagen types in the pelvic floor. They are of 
vital importance for maintaining the elasticity and toughness of the pelvic floor support system. To reveal the effect of estradiol on ECM, we treated L929 fibroblasts according to the cell experiment design, after which the ECM production of L929 fibroblast was investigated by Western blotting and Q-PCR. As can be seen from Fig. $2(a-c)$ and Fig. $3(a-c)$, both the protein and mRNA expressions of COL1A1, COL3A1, and elastin were dramatically improved in the group E2 cells when compared with those in group $C$ cells. Similarly, both the protein and mRNA expressions of COL1A1, COL3A1, and elastin were also dramatically improved in the group S+E2 cells when compared with those in the group $S$ cells. These data together suggest that moderate $\beta$-estradiol has a positive effect on the expression of ECM.

\subsection{Estradiol induces TIMP/MMP imbalance on L929 fibroblasts}

Under normal physiological conditions, MMPs and TIMPs maintain a state of dynamic balance, which is essential for maintaining ECM homeostasis. MMPs mainly hydrolyze collagen and the basement membrane, while TIMPs inhibit the breakdown of ECM and the basement membrane. We conducted western blotting and Q-PCR to investigate the expression of MMP-2, MMP-9, TIMP-1, and TIMP-2 in the L929 fibroblasts among the four groups. As shown in Fig. $2(\mathrm{~d}-\mathrm{g})$ and Fig. $3(\mathrm{~d}-\mathrm{g})$, both the protein and mRNA expressions of MMP-2 and MMP-9 were significantly reduced in the group E2 cells when compared with that in the group $C$ cells. Both the protein and mRNA expressions of TIMP-1 and MMP-9 showed dramatic improvement in the group S+E2 cells relative to that in the group $S$ cells. These data together suggest that moderate $\beta$-estradiol can increase the expression of MMPs while decreasing the expression of TIMPs, thereby maintaining a balance of TIMP/MMP to stabilize the ECM homeostasis.

\subsection{Estradiol improved the urodynamic parameters of LPP and ALPP in female SUI mice}

To further corroborate the results of the above-mentioned in vitro studies, we employed the vaginal dilation method for establishing a mouse model of SUI disease. The computer, physiological recorder, pressure sensor, micro-injection pump, and other devices were used to ascertain that the urodynamic parameters were connected to obtain a urodynamic curve. For instance, the LPPS and ALPPs of mice in groups $C$ and E2 are shown in Table 2. The LPPS and ALPPs of mice in groups VD and VD+E2 are shown in Table 3. The quantitative analyses of the parameters are indicated in Tables 2 and 3. As can be seen from these tables, the levels of LPPS and ALPPs were almost identical between groups $C$ and E2. However, the LPPs and ALPPs in the group VD+E2 were significantly higher than those in the group VD. These data suggest that, for female mice with SUI, vaginal topical application of estrogen can improve the symptoms of urinary incontinence, although, for normal mice, the vaginal topical application of estrogen showed no significant effect on the assessed urodynamic parameters. 
Table 2

Comparison of the urodynamic parameters between Group C and Group E2 ( $\mathrm{n}=$ 15)

\begin{tabular}{|llllll|}
\hline Index & Group C & Group E2 & Difference(E2-C) & $\mathbf{t}$ & $\mathbf{P}$ \\
\hline LPP $(\mathrm{mmHg})$ & $20.04 \pm 3.07$ & $21.59 \pm 3.22$ & $1.43 \pm 1.15$ & 1.26 & 0.22 \\
\hline ALPP $(\mathrm{mmHg})$ & $21.12 \pm 2.98$ & $23.23 \pm 3.34$ & $2.10 \pm 1.16$ & 1.82 & 0.08 \\
\hline
\end{tabular}

Table 3

Comparison of the urodynamic parameters between Group VD and Group VD+E2 $(n=15)$

\begin{tabular}{|llllll|}
\hline Index & Group VD & Group VD+E2 & Difference(VD+E2-VD) & $\mathbf{t}$ & P \\
\hline LPP $(\mathrm{mmHg})$ & $13.68 \pm 2.91$ & $18.42 \pm 3.21$ & $4.73 \pm 1.12$ & 4.24 & $<0.05$ \\
\hline ALPP $(\mathrm{mmHg})$ & $15.58 \pm 3.14$ & $20.33 \pm 2.98$ & $4.84 \pm 1.18$ & 4.26 & $<0.05$ \\
\hline
\end{tabular}

\subsection{The effect of estrogen on the remodeling of ECM in the anterior vaginal wall and the urethra of SUI mice}

To reveal the therapeutic effect of estrogen on female SUI mice, we collected samples from the urethra and anterior vaginal wall of each group of mice to investigate the expression of ECM-related genes by employing western blotting and Q-PCR. As shown in Fig. $4(\mathrm{a}-\mathrm{c})$ and Fig. $5(\mathrm{a}-\mathrm{c})$, both the protein and mRNA expressions of COL1A1, COL3A1, and elastin were dramatically enhanced in the group E2 mice when compared with those in the group $C$ mice. Analogously, both the protein and mRNA expressions of COL1A1, COL3A1, and elastin were dramatically enhanced in the group VD+E2 mice relative to those in the group VD mice. As shown in Fig. $4(\mathrm{~d}-\mathrm{g})$ and Fig. $5(\mathrm{~d}-\mathrm{g})$, the protein and mRNA expressions of MMP-2 and MMP-9 in group E2 mice were greatly reduced when compared to those in the group $C$ mice. Analogously, the protein and mRNA expressions of MMP-2 and MMP-9 in the group VD+E2 mice were significantly lower when compared to the group VD mice. As for TIMP-1 and TIMP-2, their protein and mRNA expressions were remarkably higher in group E2 mice than in the group $\mathrm{C}$ mice. Finally, both the protein and mRNA expressions of TIMP-1 and TIMP-2 were significantly higher in group VD+E2 mice than in group VD mice. These data together suggest that the effect of estrogen on the remodeling of ECM in the urethra and the anterior vaginal wall of female SUI mice is similar to the effect of estradiol on the remodeling of ECM secreted by L929 fibroblasts.

\subsection{Histological observation on the effect of estrogen on ECM remodeling of the urethra and anterior vaginal wall in}




\section{SUI mice}

In order to further reveal the effect of estrogen on the remodeling of ECM in the pelvic floor of mice with SUI, samples from the anterior vaginal wall and the urethra of inanimate mice were subjected to Masson staining and then photography (images shown in Fig. 6a-d). In these sections, the collagen fibers were blue and the muscle fibers were red. The mean optical density (MOD) value obtained by Image $J$ was regarded as the quantitative index to assess the collagen fibers' content, as shown in Fig. 6(e). The collagen fibers' content in the group E2 mice was significantly greater than in the group C mice. Similarly, the collagen fibers' content in the group VD+E2 mice was also significantly greater than those in the group VD mice. These data together indicated that estrogen could effectively upregulate the expression of collagen fibers, which may explain why estrogen could alleviate the symptoms of mice with SUI.

\section{Discussion}

SUI is a common disease affecting women. It reportedly results from an imbalance of ECM remodeling in the urethra and anterior vaginal ${ }^{26,29}$. ECM reconstruction is mainly regulated by fibroblasts present in the pelvic floor ${ }^{30}$. Based on the present and previous study results, we selected L929 fibroblasts as our experimental cells ${ }^{26}$. Collagen I and collagen III are the main types of pelvic floor collagen, while elastin is the key component of connective tissues that provide elasticity and support to the pelvic floor tissues ${ }^{31}$. In this study, we found that the expression of collagen I, collagen III, and elastin were lower in the urethra and anterior vaginal wall of mice with SUI when compared with those in the control group mice. Based on the above results, we obviously speculated that the restoration of the expression of ECM may facilitate SUI treatment.

McKenzie et al. [32] reported that estrogen affects the gene transcription and the expression of collagen, which is the main component of ECM ${ }^{32}$. ERT has been proven to improve certain aspects of the skin, such as by enhancing the morphology and synthesis of elastic fibers, collagen III, and hyaluronic acid to reduce wrinkles ${ }^{33}$. Past studies have demonstrated that conjugated equine estrogen can improve bone quality as it increases the amount of collagen $\mathrm{I}^{34}$. Other past studies have shown that estrogen plays a positive role in protecting glaucoma. For instance, estrogen activates the synthesis of collagen and enhances the flexibility of the eyes, thereby reducing the intraocular pressure ${ }^{35}$. Past studies reported that, in the menopausal rat model, local estrogen treatment increased the content of total collagen and crosslinked collagen, which significantly stimulated the expression of collagen mRNA in a dose-dependent manner ${ }^{36}$. Based on the above findings, we attempted to evaluate the efficacy of estrogen for the treatment of SUI mice. The results revealed that the LPP and ALPP of SUI mice treated with estrogen were higher than those of the control mice. Moreover, the expression of the urethra and the anterior vaginal wall in SUI mice treated with estrogen. The urethra and anterior vaginal wall of SUI mice expressed higher levels of collagen I, collagen III, elastin, TIMP-1, and TIMP-2 and lower levels of MMP-2 and MMP-9 relative to those of the control group mice. Our in vitro experiments revealed the same outcomes, both L929 fibroblasts after cyclic loading and L929 fibroblasts without cyclic loading expressed higher levels 
of collagen I, collagen III, elastin, TIMP-1, and TIMP-2 and lower levels of MMP-2 and MMP-9 in the presence of estrogen when compared with fibroblasts without estrogen treatment. These results together suggest that estrogen may alleviate the symptoms of SUI by reconstituting ECM.

The guidelines toward the diagnosis and treatment of female SUI (2017) in China indicated that vaginal local estrogen therapy can relieve some postmenopausal SUI symptoms and lower urinary tract symptoms. However, the reason why estrogen can be used to treat female SUI continues to remain unclear, and past studies have not been systematic in this regard. Although this study confirmed the effect of estrogen on ECM remodeling for the treatment of female SUI, the specific mechanism remains to be explored. The classic pathway of estrogen action is the estrogen receptor (ER) pathway. Past studies have demonstrated that $17 \beta$-estradiol treatment in cells can activate $\mathrm{ER}^{37}$. The ER subtypes mainly include $E R a$ and $E r \beta$, and past studies have indicated that, in cardiac fibroblasts, $17 \beta$-estradiol has a sexspecific activation effect on $\mathrm{ERa}$ and $\operatorname{Er} \beta^{38}$. Moreover, past studies have demonstrated that, in skin fibroblasts, $17-\beta$ estradiol can significantly reduce the stimulation of TGF- $\beta$ on collagen synthesis as well as reduce the activation of the classic TGF- $\beta /$ Smad $2 / 3$ signaling pathway ${ }^{39}$. We thus speculated that estrogen may have other pathways to mediate ECM remodeling, and plan to focus on the same in our future work.

\section{Declarations}

\section{Acknowledgements}

The present study was supported by the National Natural Science Foundation of China (grant Nos. 81971364 and 81771562), second level fund of the second medical leading talents project of Hubei province (No. [2019]47), and the National Key Research and Development Program of China (2018YFC2002204).

\section{Conflict of Interest}

The authors have declared that they have no conflict of interest.

\section{Author contributions}

LL: study design, data curation, formal analysis, and manuscript writing; SS: protocol development and data analysis; YL: cell experiment, data collection, and analysis ;XL: mouse modeling, project administration,data collection, and analysis; JF: tissue sampling of mice and data collection; LY:molecular experiment, data collection, and analysis; LH: conceptualization, funding acquisition, and final approval.

\section{Ethics approval}

All our animal experimental protocols and implementations were conducted based on the institutional guidelines of the Institutional Review Committee and authorized by the Ethics Committee of the 
Institutional Animal Care and Use Committee of Wuhan University People's Hospital (20210306).

\section{Data Availability Statement}

The data that support the findings of this study are available from the corresponding author upon reasonable request.

\section{References}

1. Kadekawa K, Kawamorita N, Shimizu T, et al. Effects of a selective androgen receptor modulator (SARM), GSK2849466A, on stress urinary incontinence and bladder activity in rats with ovariectomyinduced oestrogen deficiency. BJU Int. 2020;125:911-9.

2. Lo TS, Al-Kharabsheh AM, Pue LB, Ng KL, Huang TX. Mid Urethral Slings for the Treatment of Urodynamic Stress Incontinence in Overweight and Obese Women: Surgical Outcomes and Preoperative Predictors of Failure. J Urol. 2020;204:787-92.

3. Abufaraj M, Xu T, Cao C, et al. Prevalence and Trends in Urinary Incontinence Among Women in the United States 2005-2018. Am J Obstet Gynecol. 2021; 225:166.e1-e12.

4. Nygaard IE, Wolpern A, Bardsley T, Egger MJ, Shaw JM. Early postpartum physical activity and pelvic floor support and symptoms 1 year postpartum. Am J Obstet Gynecol. 2021;224:191-13.

5. Ling C, Shek KL, Gillor M, Caudwell-Hall J, Dietz HP. Is location of urethral kinking a confounder of association between urethral closure pressure and stress urinary incontinence? Ultrasound Obstet Gynecol. 2021;57:488-92.

6. Kadekawa K, Kawamorita N, Shimizu T, et al.. Effects of a selective androgen receptor modulator (SARM), GSK2849466A, on stress urinary incontinence and bladder activity in rats with ovariectomyinduced oestrogen deficiency. BJU Int. 2020;125:911-9.

7. Augoulea A, Sioutis D, Rizos D, et al.. Stress urinary incontinence and endogenous sex steroids in postmenopausal women. Neurourol Urodyn. 2017;36:121-5.

8. Melville JL, Fan MY, Newton K, Fenner D. Fecal incontinence in US women: a population-based study. Am J Obstet Gynecol. 2005;193:2071-6.

9. Chen B, Yeh J. Alterations in connective tissue metabolism in stress incontinence and prolapse. $J$ Urol. 2011;186:1768-72.

10. Jiao R, Liu Y, Liu B, Liu Z. Risk factors related to acupuncture response in postmenopausal women with stress urinary incontinence: Secondary analysis of a randomized controlled trial. Medicine (Baltimore). 2019;98:e15220.

11. Hwang UJ, Lee MS, Jung SH, Ahn SH, Kwon OY. Effect of pelvic floor electrical stimulation on diaphragm excursion and rib cage movement during tidal and forceful breathing and coughing in women with stress urinary incontinence: A randomized controlled trial. Medicine (Baltimore). 2021;100:e24158. 
12. Dumoulin C, Morin M, Danieli C, et al. Group-Based vs Individual Pelvic Floor Muscle Training to Treat Urinary Incontinence in Older Women: A Randomized Clinical Trial. JAMA Intern Med. 2020;180:128493.

13. Russo E, Caretto M, Giannini A, et al. Management of urinary incontinence in postmenopausal women: An EMAS clinical guide. Maturitas. 2021;143:223-30.

14. Faioli R, Sozzi G, Chiantera V, et al. Anterior/Apical single incision mesh (Elevate): Surgical experience, anatomical and functional results, and long-term complications. Eur J Obstet Gynecol Reprod Biol. 2021;260:166-70.

15. Goessens E, Deriemaeker H, Cammu H. The Use of a Vaginal Pessary to Decide Whether a Mid Urethral Sling should be Added to Prolapse Surgery. J Urol. 2020;203:598-603.

16. Long CY, Lin KL, Shen CR, et al. A pilot study: effectiveness of local injection of autologous plateletrich plasma in treating women with stress urinary incontinence. Sci Rep. 2021;11:1584.

17. Peyronnet B, Capon G, Belas O, et al. Robot-assisted AMS-800 Artificial Urinary Sphincter Bladder Neck Implantation in Female Patients with Stress Urinary Incontinence. Eur Urol. 2019;75:169-75.

18. Li Y, Zhang QY, Sun BF, et al. Single-cell transcriptome profiling of the vaginal wall in women with severe anterior vaginal prolapse. Nat Commun. 2021;12:87.

19. Li Y, Li BS, Liu C, et al. Effect of integrin beta1 in the treatment of stress urinary incontinence by electrical stimulation. Mol Med Rep. 2019;19:4727-34.

20. McKenzie P, Rohozinski J, Badlani G. Genetic influences on stress urinary incontinence. Curr Opin Urol. 2010;20:291-5.

21. Love MR, Palee S, Chattipakorn SC, Chattipakorn N. Effects of electrical stimulation on cell proliferation and apoptosis. J Cell Physiol. 2018;233:1860-76.

22. Richmond CF, Martin DK, Yip SO, Dick MA, Erekson EA. Effect of Supervised Pelvic Floor Biofeedback and Electrical Stimulation in Women With Mixed and Stress Urinary Incontinence. Female Pelvic Med Reconstr Surg. 2016;22:324-7.

23. Xu H, Liu B, Wu J, et al.. A Pilot Randomized Placebo Controlled Trial of Electroacupuncture for Women with Pure Stress Urinary Incontinence. PLoS One. 2016;11:e150821.

24. Cody JD, Jacobs ML, Richardson K, Moehrer B, Hextall A. Oestrogen therapy for urinary incontinence in post-menopausal women. Cochrane Database Syst Rev. 2012;10:D1405.

25. Li Y, Li BS, Liu C, et al. Effect of integrin beta1 in the treatment of stress urinary incontinence by electrical stimulation. Mol Med Rep. 2019;196:4727-34.

26. Tang J, Li B, Liu C, et al. Mechanism of Mechanical Trauma-Induced Extracellular Matrix Remodeling of Fibroblasts in Association with Nrf2/ARE Signaling Suppression Mediating TGF-beta1/Smad3 Signaling Inhibition. Oxid Med Cell Longev. 2017;2017:8524353.

27. Min J, Li B, Liu C, et al. Extracellular matrix metabolism disorder induced by mechanical strain on human parametrial ligament fibroblasts. Mol Med Rep. 2017;15:3278-84. 
28. Montoya TI, Maldonado PA, Acevedo JF, Word RA. Effect of vaginal or systemic estrogen on dynamics of collagen assembly in the rat vaginal wall. Biol Reprod. 2015;92:43.

29. Harten IA, Evanko SP, Choe $\mathrm{CH}$, et al. The extracellular matrix molecules versican and hyaluronan in urethral and vaginal tissues in stress urinary incontinence. Neurourol Urodyn. 2021;40:771-82.

30. Sun X, Zhu H, Li W, et al. Small extracellular vesicles secreted by vaginal fibroblasts exert inhibitory effect in female stress urinary incontinence through regulating the function of fibroblasts. PLoS One. 2021;16:e249977.

31. Kerkhof MH, Hendriks L, Brolmann HA. Changes in connective tissue in patients with pelvic organ prolapse--a review of the current literature. Int Urogynecol J Pelvic Floor Dysfunct. 2009;20:461-74.

32. Min J, Li B, Liu C, et al. Extracellular matrix metabolism disorder induced by mechanical strain on human parametrial ligament fibroblasts. Mol Med Rep. 2017;15:3278-84.

33. Shu YY, Maibach HI. Estrogen and skin: therapeutic options. Am J Clin Dermatol. 2011;12:297-311.

34. Condi FL, Soares JJ, Teodoro WR, et al. The effects of conjugated estrogen, raloxifene and soy extract on collagen in rat bones. Climacteric. 2012;15:441-8.

35. Wei X, Cai SP, Zhang X, Li X, Chen X, Liu X. Is low dose of estrogen beneficial for prevention of glaucoma? Med Hypotheses. 2012;79:377-80.

36. Montoya TI, Maldonado PA, Acevedo JF, Word RA. Effect of vaginal or systemic estrogen on dynamics of collagen assembly in the rat vaginal wall. Biol Reprod. 2015;92:43.

37. Hosford SR, Shee K, Wells JD, et al. Estrogen therapy induces an unfolded protein response to drive cell death in ER+ breast cancer. Mol Oncol. 2019;13:1778-94.

38. Dworatzek E, Mahmoodzadeh S, Schriever C, et al. Sex-specific regulation of collagen I and III expression by 17beta-Estradiol in cardiac fibroblasts: role of estrogen receptors. Cardiovasc Res. 2019;115:315-27.

39. Avouac J, Pezet S, Gonzalez V, et al. Estrogens Counteract the Profibrotic Effects of TGF-beta and their Inhibition Exacerbates Experimental Dermal Fibrosis. . 2020;140:593-601.

\section{Figures}




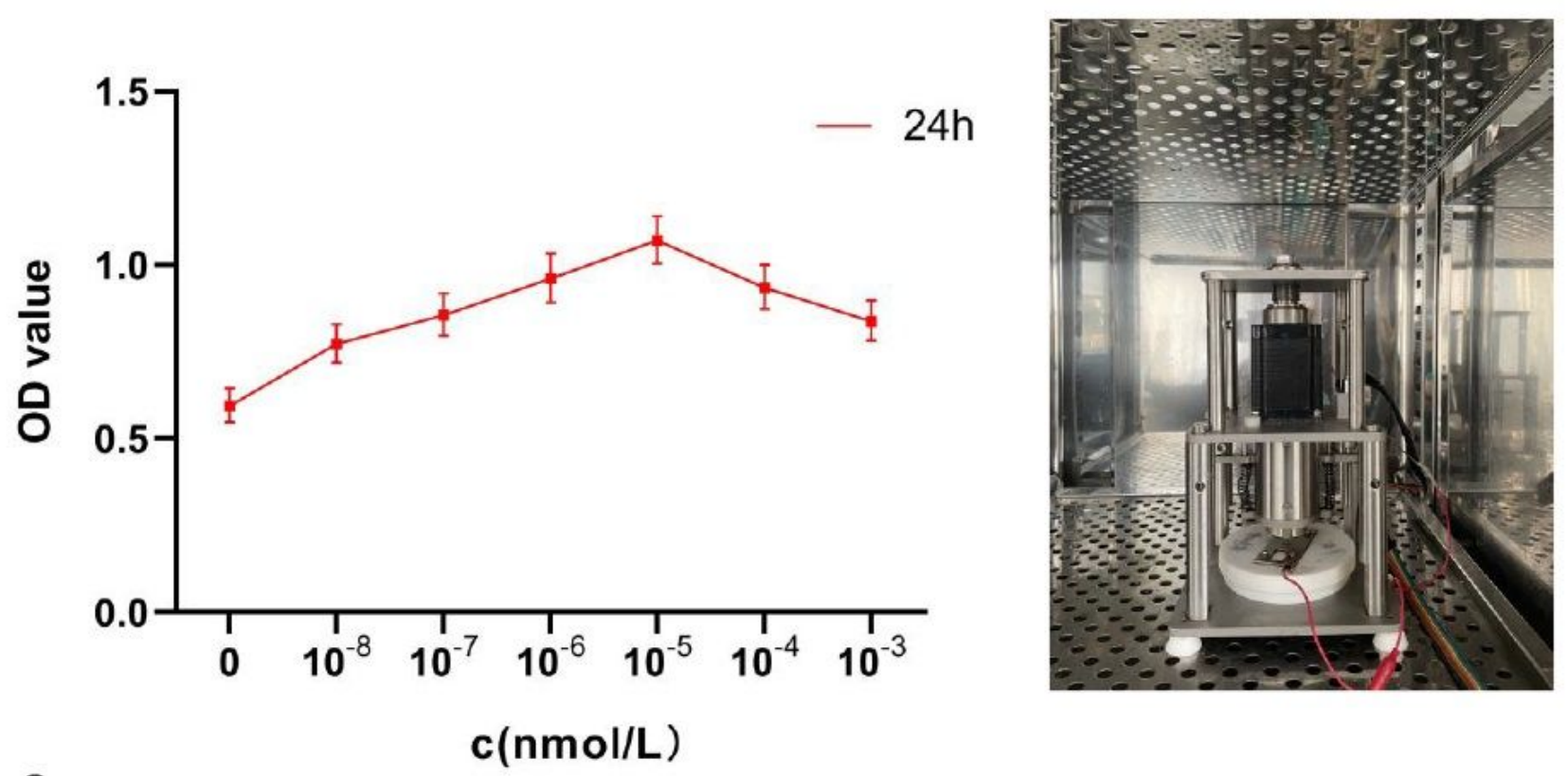

C

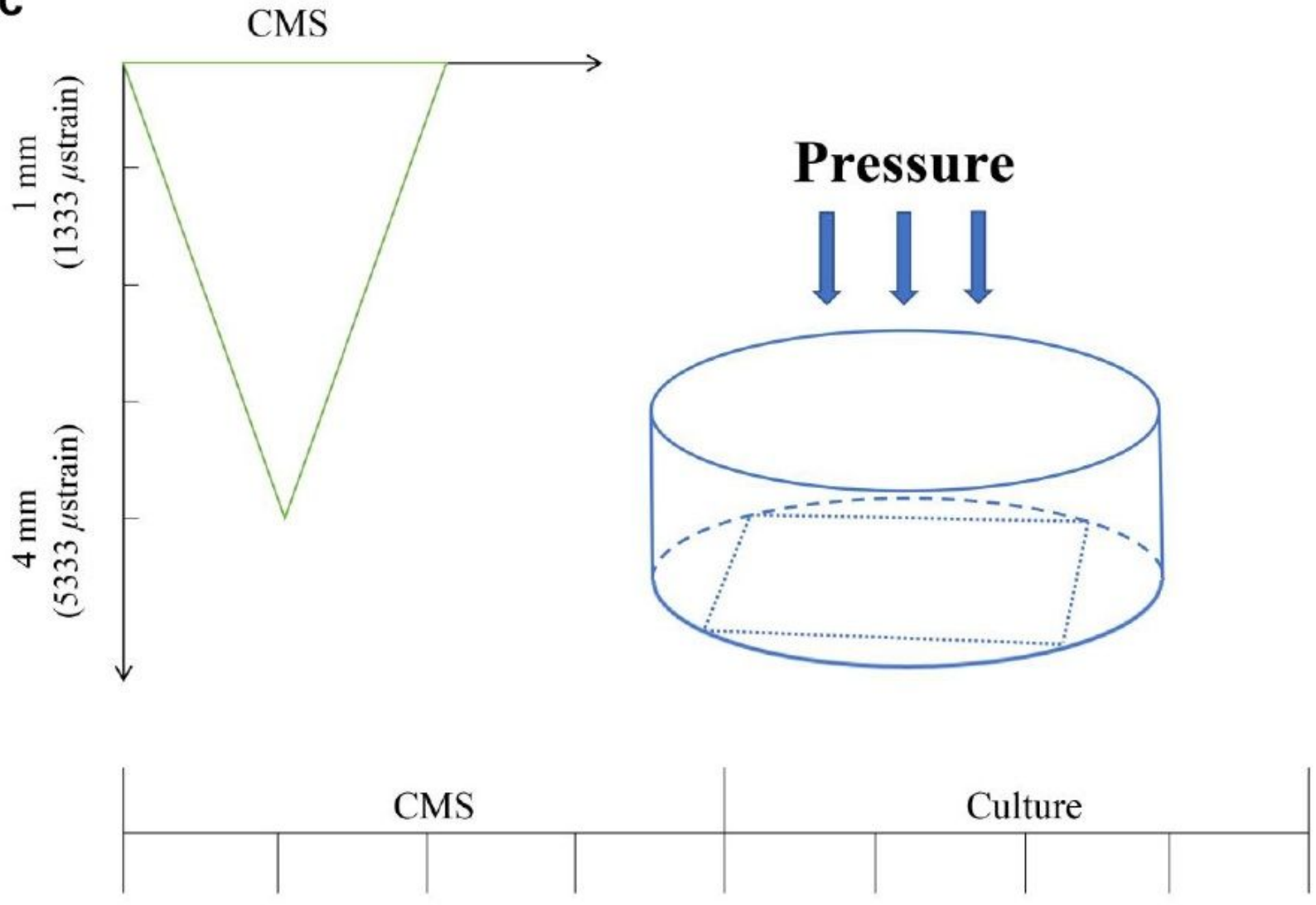

Figure 1

The cyclic mechanical strain on L929 fibroblasts. (a) The proliferation of L929 cells with different concentrations of estradiol was performed with the Cell Counting Kit-8. (b) The strain gauges and fourpoint bending cell strain gauges. (c) Experimental diagram and the simulation diagram of mechanical strain. 


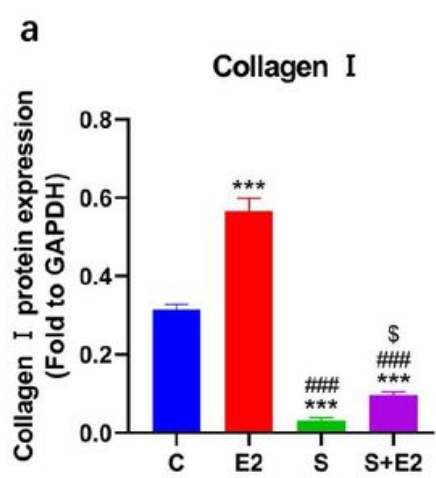

e

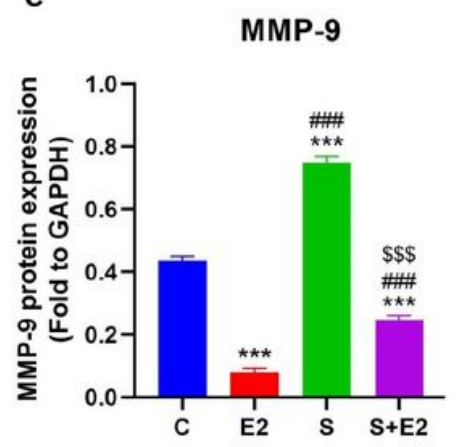

b

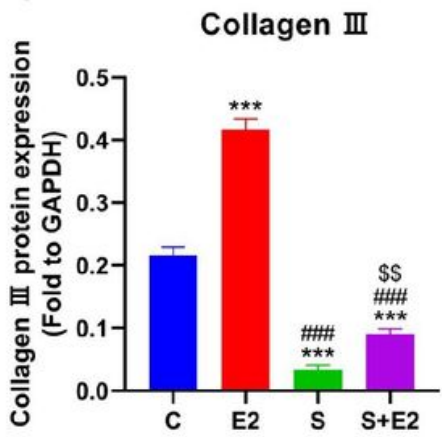

f

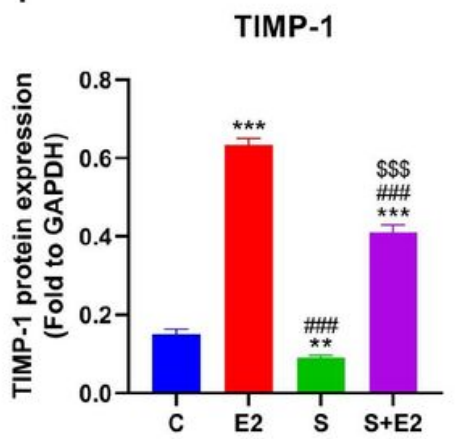

c

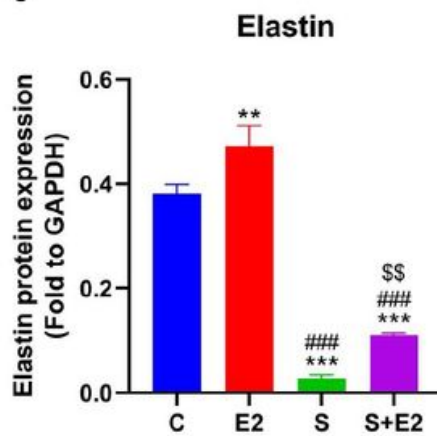

g

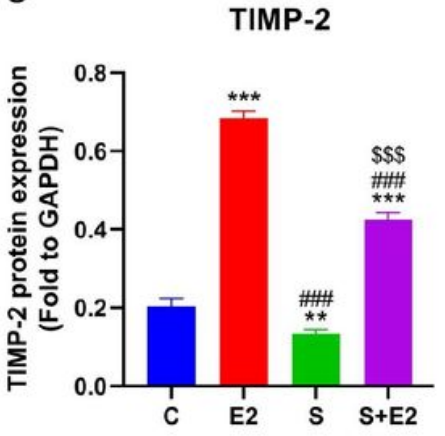

d

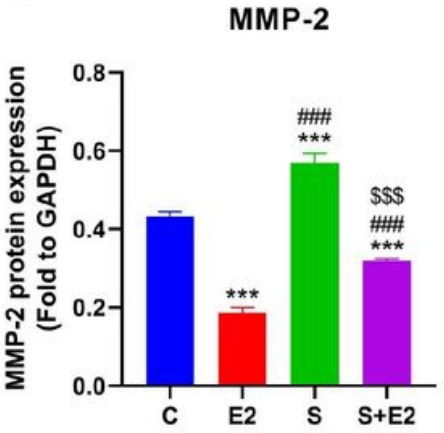

h

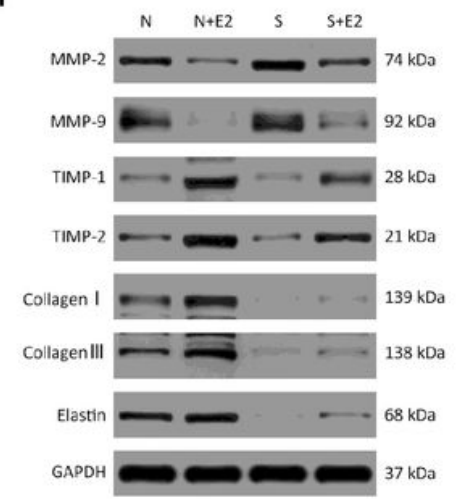

\section{Figure 2}

The promoting effect of estradiol on the ECM expression for L929 fibroblasts. (a-c) Estradiol promoted

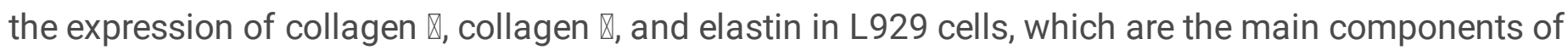
ECM. (d-e) Estradiol inhibited the expression of MMP-2 and MMP-9 in L929 cells. ( $f-g)$ Estradiol promoted the expression of TIMP-1 and TIMP-2 in L929 cells. (h) Protein electrophoresis bands. (C: normal cells without estrogen therapy; E2: normal cells with estrogen therapy; $\mathrm{S}$ : cells undergone cyclic mechanical strain without estrogen therapy; $\mathrm{S}+\mathrm{E} 2$ : cells undergone cyclic mechanical strain with estrogen

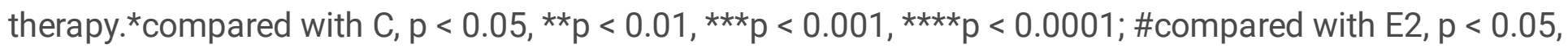
$\# \# p<0.01, \# \# \# p<0.001, \# \# \# \# p<0.0001 ;$ \$compared with $\mathrm{S}, \mathrm{p}<0.05$,

$$
p<0.01,
$$

$\$ p<0.001$,

$p<0.0001$. Every experiment was repeated thrice). 


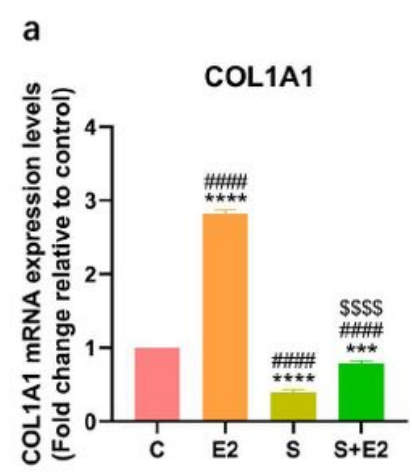

e

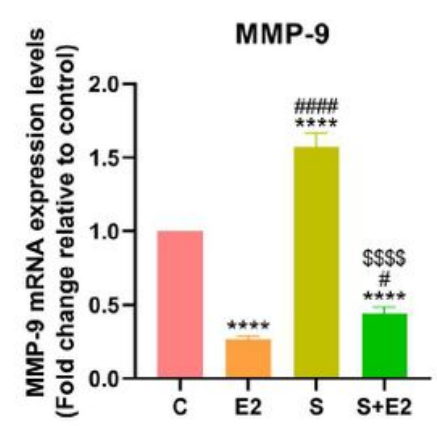

b
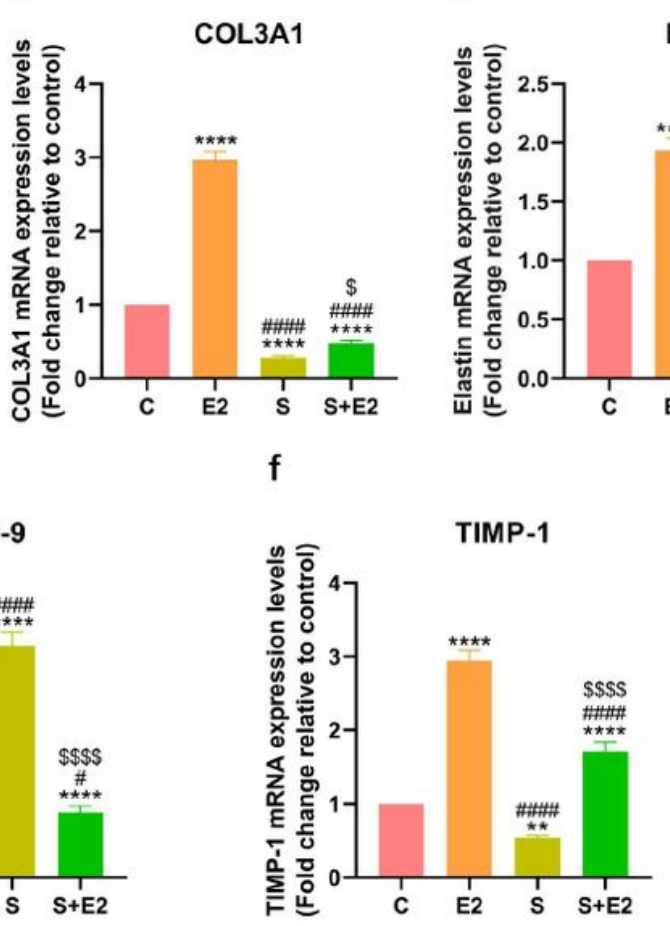

d
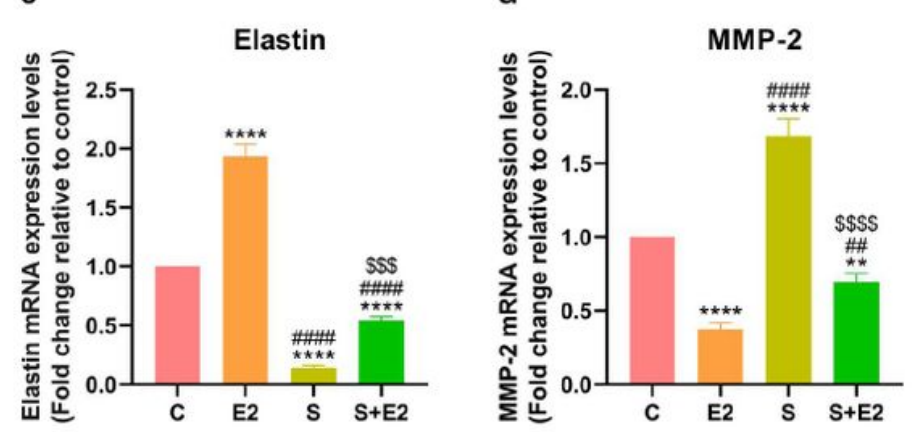

g

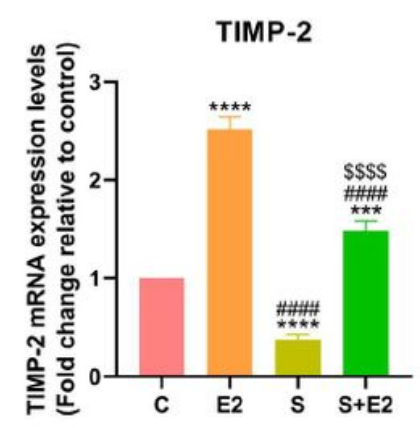

\section{Figure 3}

Estradiol promotes the mRNA expression levels of collagen $\nabla$, collagen $\nabla$, and elastin related to the ECM in

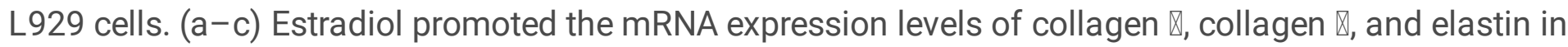
L929 cells. ( $d-e)$ Estradiol inhibited the mRNA expression levels of MMP-2 and MMP-9 in L929 cells. (fg) Estradiol promoted the mRNA expression levels of TIMP-1 and TIMP-2 in L929 cells. (C: normal cells without estrogen therapy; E2: normal cells with estrogen therapy; S: cells that underwent cyclic mechanical strain without estrogen therapy; S+E2: cells that underwent cyclic mechanical strain with estrogen therapy. ${ }^{*}$ compared with $\mathrm{C}, \mathrm{p}<0.05$, ${ }^{*} \mathrm{p}<0.01$, ${ }^{* \star} \mathrm{p}<0.001$, ${ }^{\star \star * *} \mathrm{p}<0.0001$; \#compared with E2, $p<0.05, \# \# p<0.01, \# \# \# p<0.001, \# \# \# \# p<0.0001$; \$compared with $S, p<0.05$,

$$
p<0.01 \text {, }
$$

$\$ p<0.001$

$p<0.0001$. Every experiment was repeated thrice). 
a

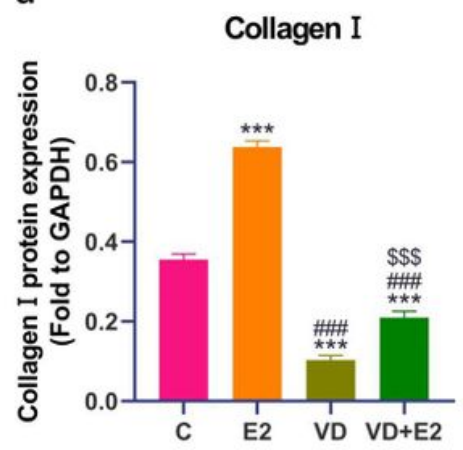

d

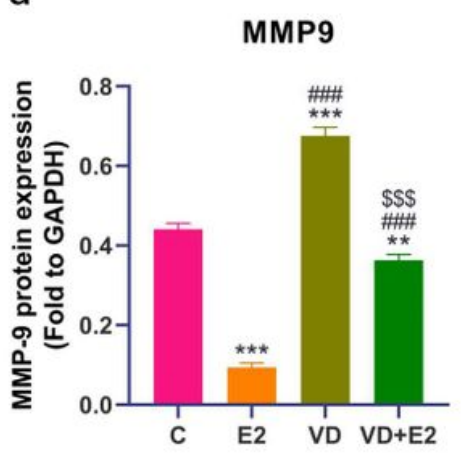

b

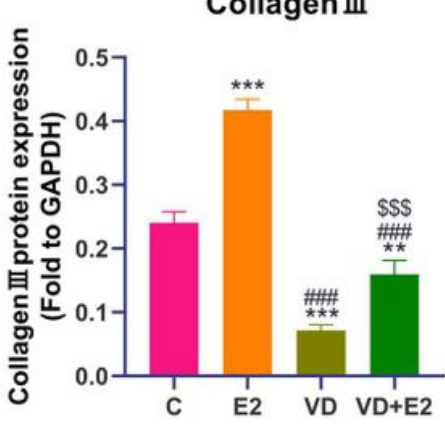

e

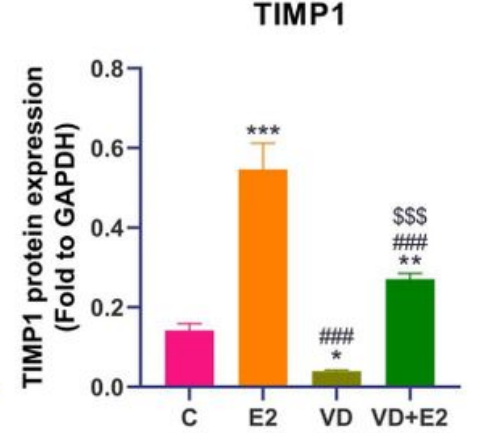

C

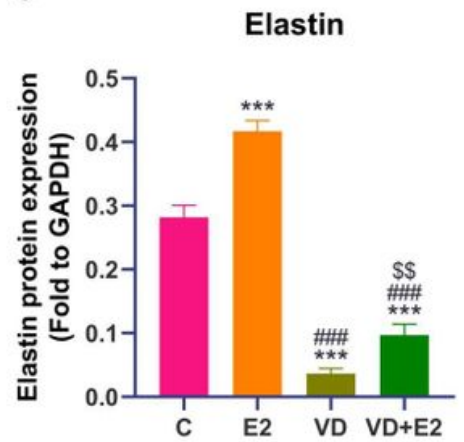

f

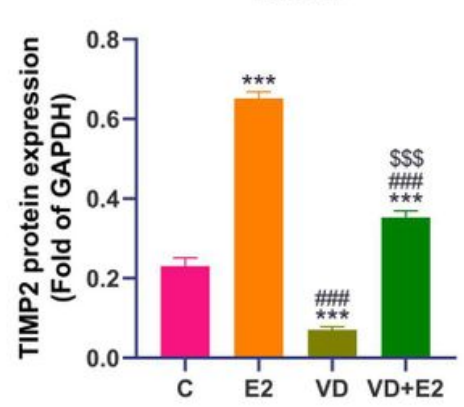

d

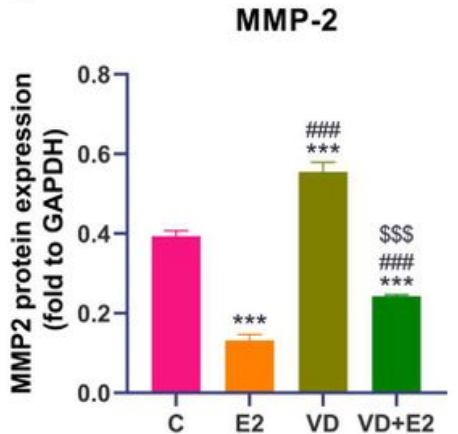

g

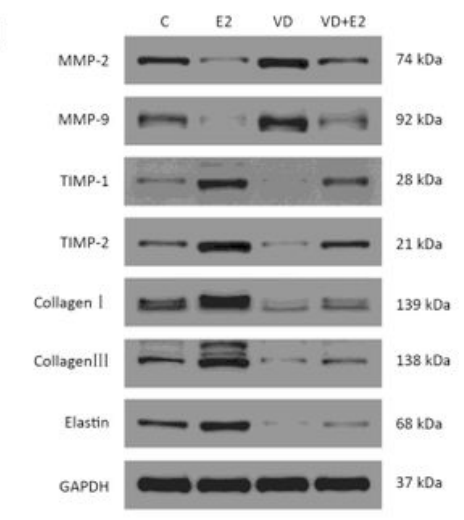

Figure 4

The effect of estrogen on ECM remodeling in the anterior vaginal wall and the urethra of SUI mice. $(a-c)$ Estradiol promoted the expression of collagen $\otimes$, collagen $\otimes$, and elastin in the vaginal wall and the urethra of SUI mice. (d-e) Estradiol inhibited the expression of MMP-2 and MMP-9 in the vaginal wall and the urethra of SUI mice. $(f-g)$ Estradiol promoted the expression of TIMP-1 and TIMP-2 in the vaginal wall and the urethra of SUI mice. (h) Protein electrophoresis bands. (C: control group; E2: estrogen-alone group; VD: vaginal dilatation group; $V D+E 2$ : vaginal dilation with the estrogen group. *compared with $C, p$ $<0.05, * * p<0.01, * * * p<0.001,{ }^{* * * *} p<0.0001$; \#compared with E2, $p<0.05, \# \# p<0.01, \# \# \# p<0.001$, $\# \# \#$ \# $<0.0001 ;$ \$compared with VD, $p<0.05$,

$$
p<0.01,
$$

$\$ p<0.001$

$p<0.0001$. Every experiment was repeated thrice). 
a

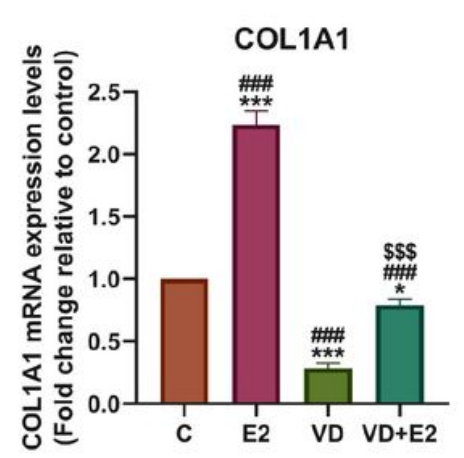

e

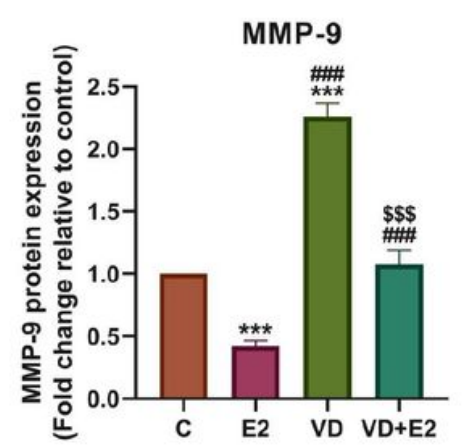

b

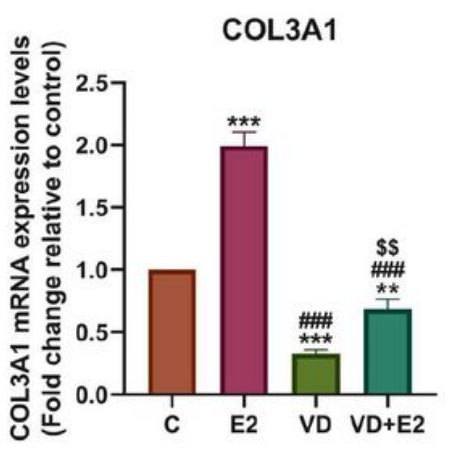

f

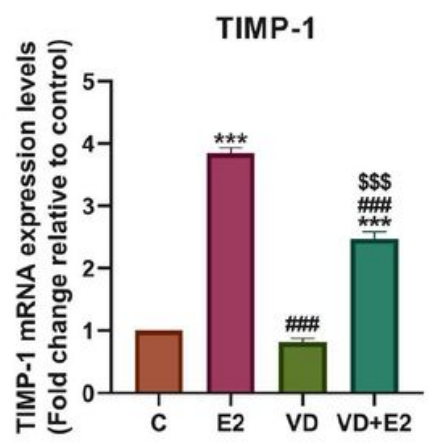

d
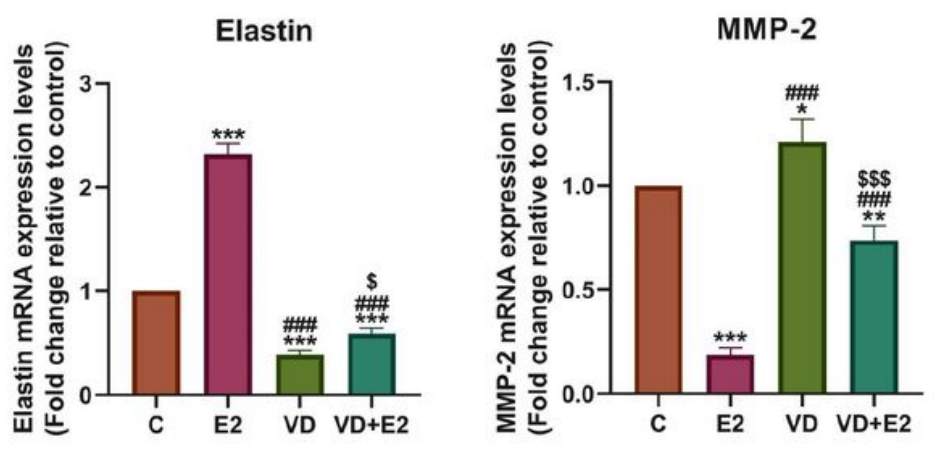

g

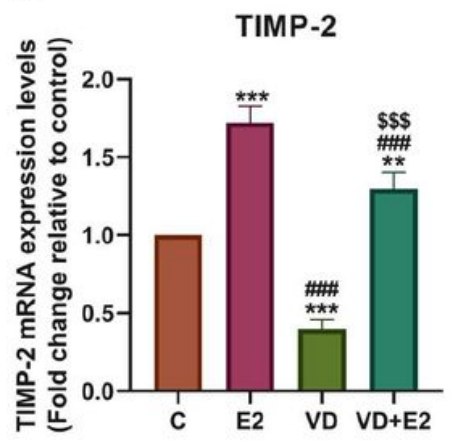

\section{Figure 5}

Estradiol promoted the mRNA expression levels of collagen $\nabla$, collagen $\nabla$, and elastin related to the ECM in the anterior vaginal wall and the urethra of SUI mice. $(\mathrm{a}-\mathrm{c})$ Estradiol promoted the mRNA expression levels of collagen $\nabla$, collagen $\nabla$, and elastin in the anterior vaginal wall and the urethra of SUI mice. (d-e) Estradiol inhibited the mRNA expression levels of MMP-2 and MMP-9 in the anterior vaginal wall and the urethra of SUI mice. $(f-g)$ Estradiol promoted the mRNA expression levels of TIMP-1 and TIMP-2 in the anterior vaginal wall and the urethra of SUI mice. (C: control group; E2: estrogen-alone group; VD: vaginal dilatation group; $V D+E 2$ : vaginal dilation with the estrogen group. ${ }^{*}$ compared with $C, p<0.05, * \star p<0.01$,

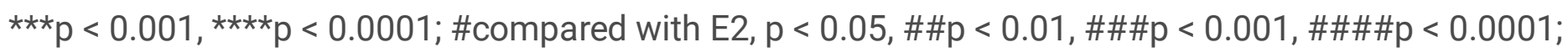
\$compared with VD, $p<0.05$,

$$
p<0.01 \text {, }
$$

$\$ p<0.001$,

$p<0.0001$. Every experiment was repeated thrice). 
a

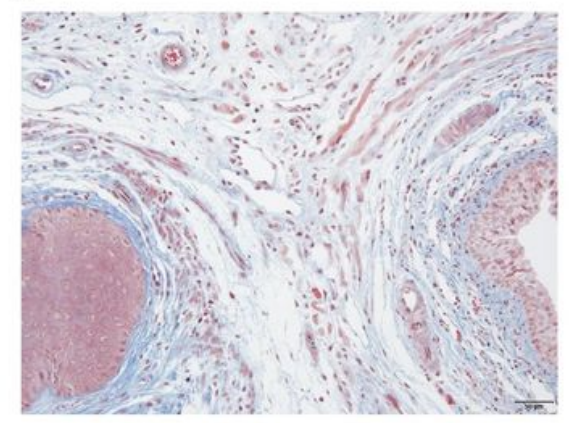

C

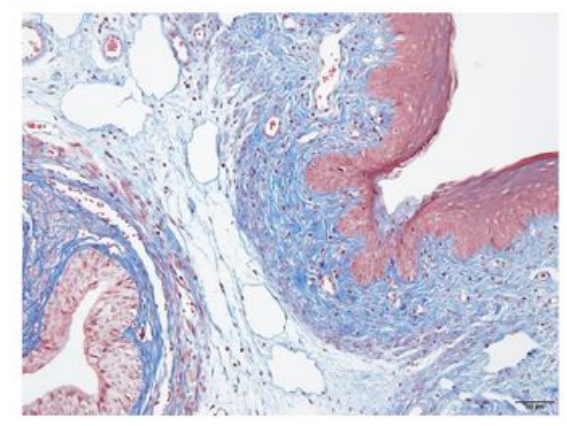

b

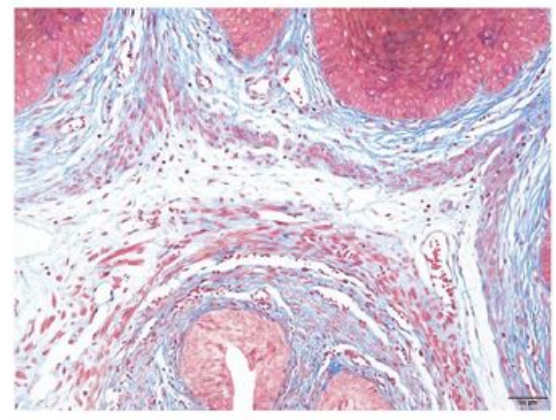

d

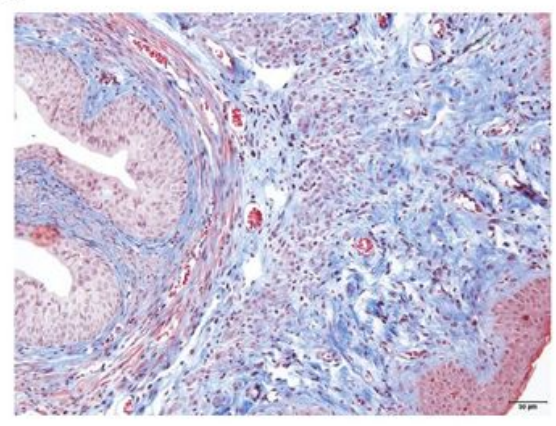

e

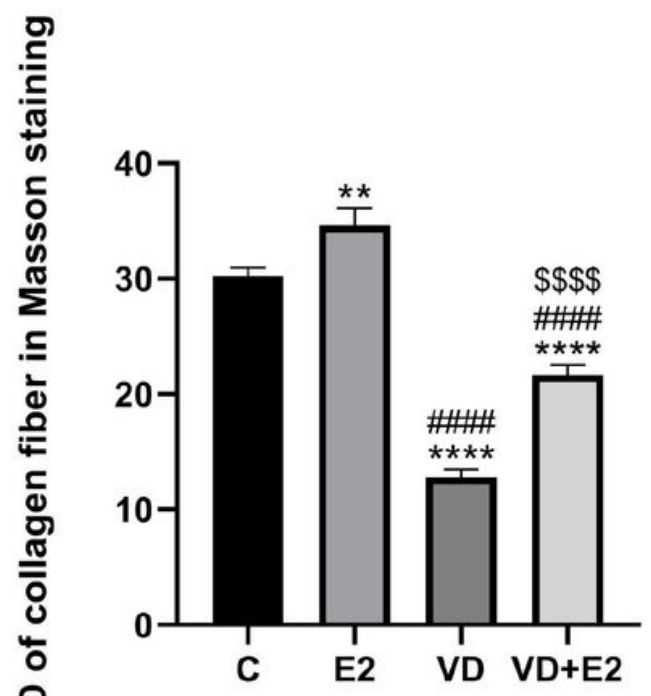

Figure 6

Histological observation of the effect of estrogen on ECM remodeling of the urethra and the anterior vaginal wall in SUI mice. (a) The collagen fibers' content in group C mice. (b) The collagen fibers' content in group E2 mice. (c) The collagen fibers' content in group VD mice. (d) The collagen fibers' content in group VD+E2 mice. (e) The MODs of collagen fibers after Masson staining. (C: control group; E2: estrogen-alone group; VD: vaginal dilatation group; VD+E2: vaginal dilation with estrogen group. *compared with $\mathrm{C}, \mathrm{p}<0.05$, ${ }^{*} \mathrm{p}<0.01$, ${ }^{\star \star *} \mathrm{p}<0.001$, ${ }^{\star \star * \star} \mathrm{p}<0.0001$; \#compared with $\mathrm{E} 2, \mathrm{p}<0.05, \# \# \mathrm{p}<$ $0.01, \# \# \# p<0.001, \# \# \# \# p<0.0001 ;$ \$compared with VD, $p<0.05$,

$$
p<0.01,
$$

$\$ p<0.001$

$\mathrm{p}<0.0001)$ 